\title{
Concept for a Large Scalable Space Telescope: In-Space Assembly
}

\author{
W. R. Oegerle, L. R. Purves, J. G. Budinoff, R. V. Moe, T. M. Carnahan, D. C. Evans, C. K. Kim \\ NASA/Goddard Space Flight Center, Greenbelt, MD 20771
}

\begin{abstract}
We present a conceptual design for a scalable (10-50 meter segmented filled-aperture) space observatory operating at UV-optical-near infrared wavelengths. This telescope is designed for assembly in space by robots, astronauts or a combination of the two, as envisioned in NASA's Vision for Space Exploration. Our operations concept for this space telescope provides for assembly and check-out in an Earth Moon L2 (EML2) orbit, and transport to a Sun-Earth L2 (SEL2) orbit for science operations and routine servicing, with return to EML2 for major servicing. We have developed and analyzed initial designs for the optical, structural, thermal and attitude control systems for a $30-\mathrm{m}$ aperture space telescope. We further describe how the separate components are packaged for launch by heavy lift vehicle(s) and the approach for the robot assembly of the telescope from these components.
\end{abstract}

Keywords: Large space telescopes, robotic assembly, orbital assembly

\section{INTRODUCTION}

The Hubble Space Telescope (HST), which operates at ultraviolet/optical/infrared (UVOIR) wavelengths of $0.115-2.5$ microns, has been the most productive scientific space telescope of all time ${ }^{1}$. HST has provided key discoveries in many areas of astrophysical research, including the nature of massive black holes, quasars, dark energy, galaxy evolution, the intergalactic medium, and planetary disks, and has has excited the general public with its stunning, high resolution views of the universe. Although the James Webb Space Telescope (JWST), slated for launch in 2013, is expected to provide tremendous new capabilities in the near infrared ${ }^{2}$, there are no plans for a future large-aperture general-purpose observatory operating at UV-optical wavelengths until at least 2030. Given that JWST will have an aperture diameter of 6.5 meters, and future ground-based telescopes operating at that time will have apertures of 20-30+ meters, we consider a strawman aperture of 30 meters for the next UVOIR space telescope. We hereafter refer to this observatory as the Thirty Meter Space Telescope (TMST). The concept for this telescope grew from ideas put forward at the Hubble Science Legacy meeting ${ }^{1}$ in 2002, and in a proposed Vision mission by Illingworth et al ${ }^{3}$ in 2003.

The tremendous scientific capability of a $\sim 30$ meter space telescope will only be briefly mentioned here. It should be remembered that diffraction-limited imaging from the ground will not be achievable in the optical portion of the spectrum even with adaptive optics $(A O)$. In the near-IR, $A O$ will permit diffraction-limited imaging only over small fields of view ( arcseconds). A 30 meter UVOIR space telescope will make major breakthroughs in our understanding of the structure and evolution of galaxies, the formation of stars and exosolar planets, stellar populations and the distribution of mass in the universe. For instance, this telescope could resolve individual sun-like stars in galaxies as far away as the Coma cluster $(\mathrm{V}=35)$ and resolve star clusters $(10 \mathrm{pc}$ diameter) in galaxies at redshifts of $\sim 2$. TMST could detect and study $\sim 500$ exosolar planets out to a distance of $100 \mathrm{pc}$, and map the disks around proto-stars in the Orion cloud complex at sub-astronomical unit scales.

In 2004, NASA published the "Vision for Space Exploration"4 (hereafter VSE), which calls for extending our reach beyond low-earth orbit with missions to the moon and Mars, as well as creating the necessary infrastructure in space (power, communications, heavy lift launch vehicles, etc) that would enable the construction of observatories in deep space. The promise of such infrastructure enables the construction of future large space telescopes, whose assembled sizes are too large to fit into current or even future launch fairings, and cannot not be meaningfully tested on Earth. In our study of the construction of a large telescope in space, we have then assumed the existence of such infrastructure especially heavy-lift launch vehicles, and the availability of robots and astronauts for in-space assembly. 
We have considered it desirable to think in terms of an observatory architecture that is "scalable"; ie. the general architecture is the same for a 20 meter aperture as for a 50 meter aperture. For the rest of this paper, however, we will consider a point-design for a telescope having a diameter equivalent to a 30 meter unobstructed filled-aperture mirror. There are a host of trade studies that need to be undertaken when deciding on the optical, mechanical, thermal design of such an observatory. Rather than trying to optimize our design, we decided to make defensible design choices based on realistic extrapolations from current technology and show that at least one reasonable design could be built and would perform as required. Previous and current work on large space telescopes and their assembly can be found in references 5-12.

\section{OPTICAL DESIGN AND WAVEFRONT SENSING}

Inherent in our concept is that the primary is composed of many smaller mirror segments. One of the obvious advantages of such a design is that the telescope could be tested and used when only partially completed. One of the trades that must be made is that of mirror segment size. We chose to use small (1-meter) circular mirrors for their ease of manufacture and ground testing. Furthermore, these small mirrors are stiff enough that no "active" figure control is required in operation; only 7 degrees of freedom should be required (tip/tilt, axial clocking, $X$ and $Y$ decenter, piston and radius of curvature). Only 14 individual optical prescriptions are required if the mirrors are placed in 14 concentric rings (see Figure 1 below). This geometry allows us to place a segment anywhere within its prescribed ring, reducing on-orbit spares inventory and simplifying assembly and servicing. While this arrangement only has a $73 \%$ fill factor, this has a minimal effect at the image plane, and reduces segment costs significantly.

We required diffraction-limited imaging at $632 \mathrm{~nm}$, resulting in an Airy disk radius of 5 milliarcsec over a field of view of 3 arcmin. After consideration of several optical designs, we chose a through-focus (Gregorian) Ritchie-Chretien, with an $\mathrm{f} / 2$ primary, effective focal length of $932.5 \mathrm{~m}$ and total system $\mathrm{f} / \#$ of 31.1 . The diameter of the secondary mirror is $2.3 \mathrm{~m}$ and the primary-secondary spacing is $64.3 \mathrm{~m}$. The resulting plate scale is 0.22 arcsec/mm. The field-of-view contains diffraction-limited images with Strehl ratio $>0.7$.

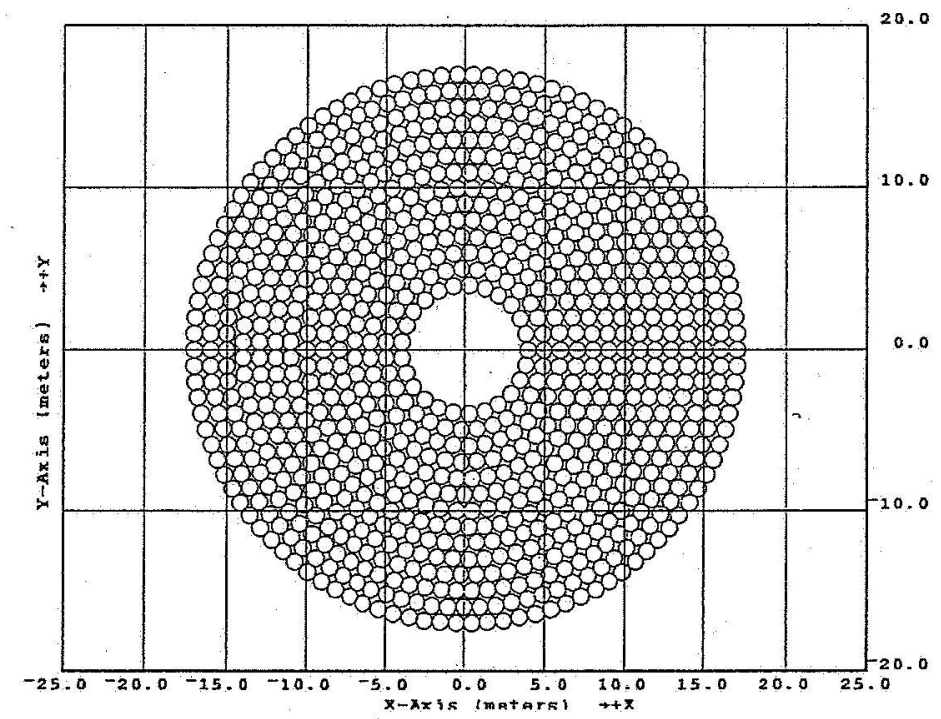

Fig 1. Primary mirror segments shown in configuration. There are 14 concentric rings of mirrors, with 916 mirrors in all.

The primary mirror segments were modeled as non-sequential elements in ZEMAX. The segment shape and irregular gaps were accurately modeled. A 3-arm secondary spider did not significantly affect the point spread function as long as the arm widths were less than $\sim 500 \mathrm{~mm}$.

There are. several different means of sensing and controlling the wavefront from these hundreds of mirror segments. Shack Hartmann sensors have been used by the ground-based Keck Observatory, and a laser metrology truss is planned 
for use with the strawman version of TPF-C. Feinup \& Dean have demonstrated that JWST can be phased using imagebased wavefront information, using only a camera. We have adopted that technique for TMST.

This phasing architecture allows the nearly 1000 segments to be phased as they are assembled. A center-of-curvature source and wavefront camera will provide the phase diversity images required for phasing. No edge sensors are required. Center of curvature architectures have not yet been implemented on ground or space observatories due to the required extra length of the metering structure. However, for in-space assembled observatories such as the TMST, this is not a limitation.

\section{OBSERVATORY DESIGN}

The optical telescope assembly (OTA) can be broken down into 4 major assemblies: the backplane, the metering tower, the sunshade, and the support truss. The OTA incorporates damping elements within the structure to reduce dynamic settling time. The secondary assembly houses the $2.3 \mathrm{~m}$ secondary mirror, its pointing/stabilization system, wavefront cameras, laser truss metrology, and a cluster of star trackers. A node and truss structure was designed to support the optics and instruments and maintain alignment stability to within the correction range of the optical controls.

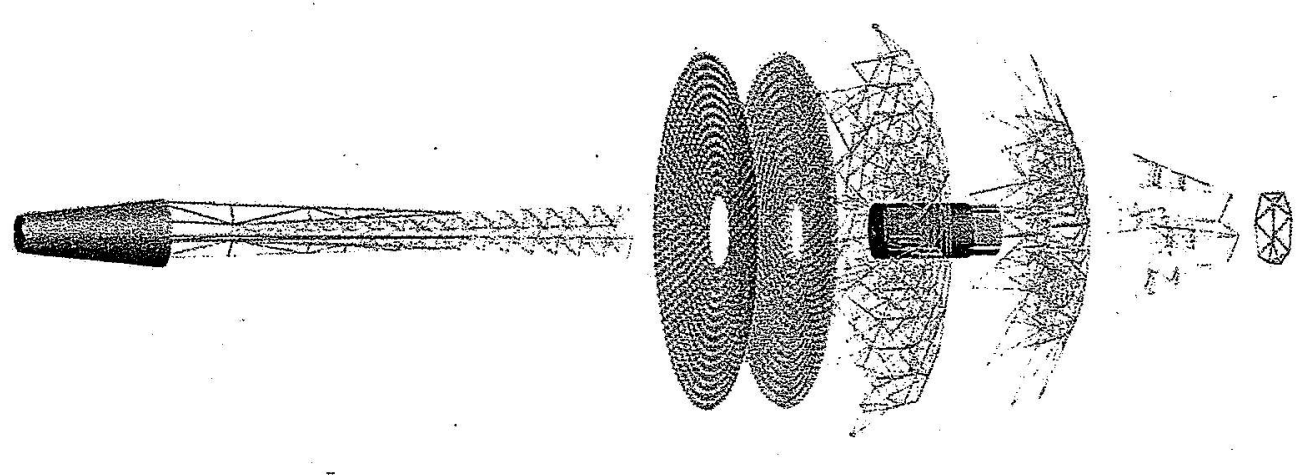

Fig 2. Exploded view of the OTA showing secondary tower, primary mirror, backplane, support truss, and ISIM.

With the optics and support structure defined, the next TMST topic that needs to be considered is the orbit or orbits in which it will be assembled, serviced and used for scientific investigations. This is because all of the remaining systems are highly influenced by this choice. The decision was made to perform TMST science operations in a Sun-Earth Lagrange-Point 2 (SEL2) orbit, which has been successfully used by WMAP and is planned for JWST. SEL2 has many advantages: (1) it is thermally stable (constant full sunlight), (2) the sun, earth and moon are all in one direction, facilitating light shielding and communications, (3) the orbit has an uninterrupted view of half the sky, and (4) it is relatively close to earth and permits relatively fast and low energy access by launch vehicles from the Earth-moon Lagrange points, where substantial infrastructure is likely to exist. Although science operations in low-earth orbit would be logistically favorable, the variable thermal and illumination environment, as well as atmospheric drag and monotomic oxygen effects, make a deep-space orbit (eg. SEL2) highly desirable. The choice of orbit then allowed us to define the rest of the observatory - the sunshade, communications, propulsion, pointing control and power system.

The resulting observatory in shown in Figure 3. The telescope optics and instruments are supported by a truss structure housed within a tubular sunshade. Outside of the sunshade is the spacecraft bus, solar arrays, communication antennae, storage/support areas for the tender robotics, and docking/berthing interfaces. The entire observatory is designed to be robotically assembled and serviced on orbit. The nominal design lifetime is 100 years with servicing.

A sunshade of some form is required to provide a constant thermal environment for the OTA and replaceable instrument modules, and to protect the optics from direct illumination by the sun. We considered a tubular light baffle like that used on HST, and a JWST-like sunshield on one side with no baffle. There are advantages and disadvantages with both 'designs; our strawman design employs a tubular light baffle. The hexagonal sunshade structure consišts of 6 deployable 
panel sheets of 15-layer insulation (MLI) and 6 triangular base closeout MLI sheets supported on a tension frame. The sunshade is supported by the bus structure and is mechanically isolated from the OTA. This closed baffle also provides some thermal control, although most of the control is performed by heaters. A daily average of $37 \mathrm{~kW}$ of power (no margin) is required to maintain the primary mirror at room temperature. Two dual-gimbaled solar arrays with area of $252 \mathrm{~m}^{2}$ and efficiency of $28 \%$ will provide $\sim 70 \mathrm{~kW}$ at beginning of life. Significant power savings could be made by running the mirrors at somewhat cooler temperatures, and would also improve performance at a wavelength of 3 microns.

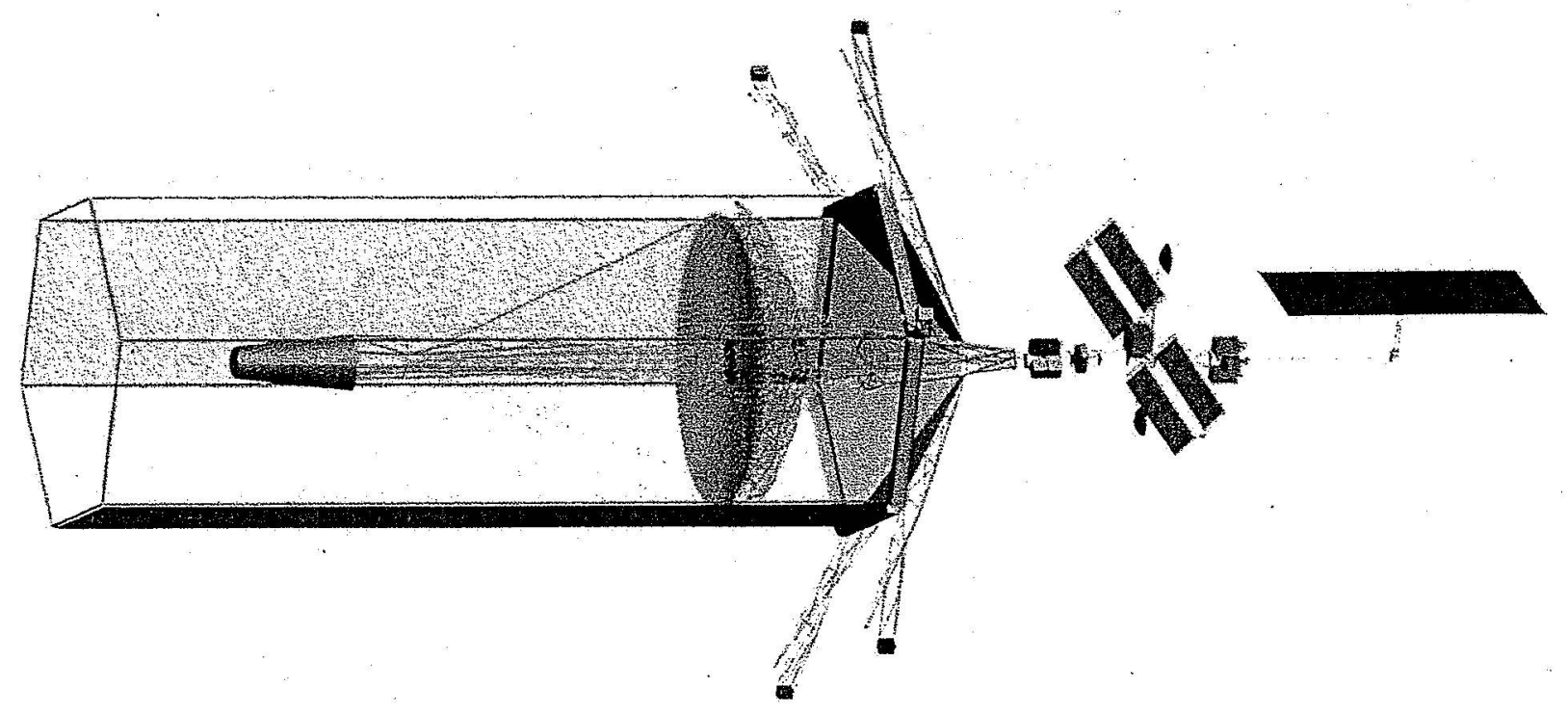

Fig 3. The TMST Observatory showing sunshade, secondary metering structure, primary mirror and backplane, spacecraft bus, solar arrays, and solar sail

The large cross-section presented by the light baffle ( 40 meters wide by over 60 meters in length) will result in significant torque due to solar photon pressure. An articulated solar sail (as shown in Figures 3 and 4) placed at the end of a long fixed boom will counter the induced torque. The boom also serves as the structural support for the other spacecraft bus subsystems. The solar sail nulls the torque due to the solar photons for any orientation of TMST. It is assumed that any remaining solar torque will be absorbed by the reaction wheels until they approach saturation, at which point the solar sail will be readjusted so that the affected wheels will begin unloading. The more precise negation of the solar torque and slewing are handled by a set of reaction wheels located near the observatory center of gravity, as shown in Figure 4. Given the size and mass of the TMST, substantial torque authority is needed for attitude control. Multiple sets of reaction wheels, based on current technology, could be built that would have the necessary torque and noise properties. Four 4-wheel pyramids (16 wheels total) where each wheel is a $400 \mathrm{Nms}, 0.5 \mathrm{Nm}$ Explorer H400 equivalent will do the job. Current star trackers and gyros are adequate for coarse pointing knowledge and control. Fine pointing corrections are provided to the attitude control system by rapid sampling of several "guide" stars in the wide-field science camera.

Another aspect of attitude control is that of jitter control and is under investigation. Structural Finite Element Analyses (FEA) have been performed on both the telescope and the other TMST elements. The FEA confirmed that the OTA (optics, instruments, and support structure) could get its lowest natural frequency mode above $1 \mathrm{~Hz}$. The FEA also showed that the other elements (sunshade, SC bus, solar sail) had natural modes in the 0.001 to $0.01 \mathrm{~Hz}$ range, which was expected. The current concept is to place an active vibration control device between the OTA and the relatively much more flexible assembly of the other TMST elements. It is possible, but unlikely, that a passive isolator, such as that planned for JWST, will be sufficient. 
SEL2 is not a stable orbit, and station-keeping (SK) maneuvers must be employed. The TMST mass and required onorbit SK maneuvers define the thrust and amount of $\Delta V$ required. As shown in Figure 4, six hydrazine monopropellant propulsion units on the sunshade booms provide backup wheel desaturation and SK attitude control. In addition, two bipropellant hydrazine/NTO transfer modules provide higher thrust for infrequent navigation maneuvers. The propulsion units are designed for 5 years of nominal operations before requiring replacement. Solar-electric ion propulsion is also an option, which was briefly looked at but needs more study. Its chief benefit would be to reduce propellant mass. Ion propulsion would most likely be practical for SEL2 SK, but a better understanding is required of the power consumption, duration of the low thrust firings, and whether science observations could continue during these maneuvers if their duration impacts observations. ELM2 is less stable than SEL2 and therefore requires higher SK accelerations, which will be more challenging with ion propulsion. Ion-propulsion might be able to support EML2 - SEL2 transfers, but study is required to see what the time impact will be.

Science data is downlinked from SEL2 to Earth at $1 \mathrm{Gbps}$ with a 4 meter aperture High Gain antenna (HGA). As shown in Fig 4, there are 2 HGAs, to allow uninterrupted data transmission regardless of TMST's orientation with respect to Earth.

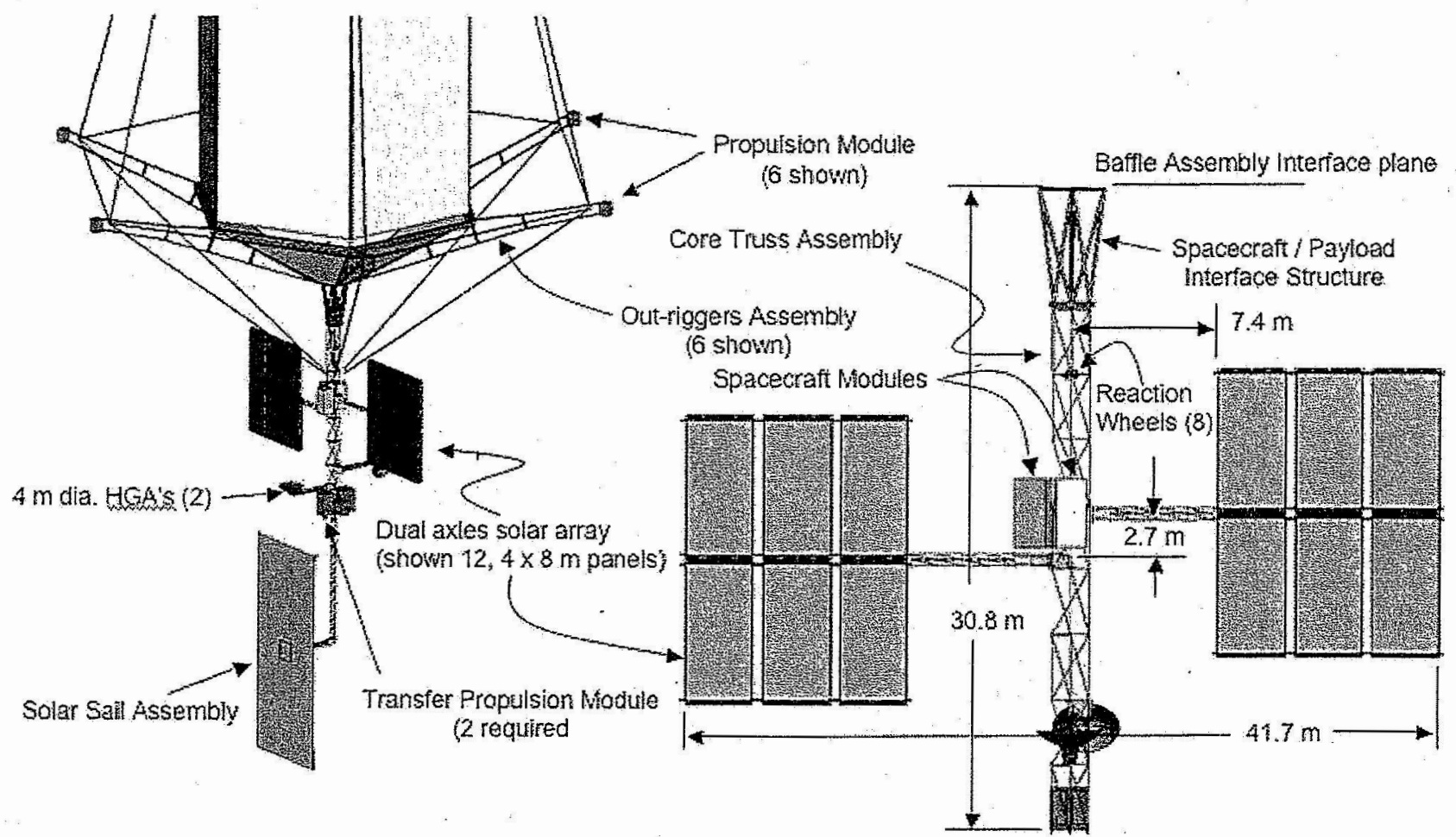

Fig 4. The picture to the left shows the lower half of the telescope (enclosed in a sunshade), with the truss extension supporting the solar arrays, high gain antennas and articulated solar sail. The picture on the right is an expanded view of the truss and spacecraft elements

\section{ASSEMBLY LOCATION AND ORBITS}

Robotic construction of an observatory at SEL2 would be challenging due to communication delays. Furthermore, there are no plans at present to station human astronauts at SEL2 to support robots. Therefore, we looked for other locations in which to assemble and test TMST before transporting it to SEL2. This study was begun prior to the announcement of the VSE, and we initially considered assembly at the International Space Station (ISS), where the infrastructure existed (power, communications, astronauts, etc). However, testing of the assembled telescope in low-earth orbit was considered problematical due to thermal and light variations on orbital timescales, and a large $\Delta \mathrm{V}(\sim 3 \mathrm{~km} / \mathrm{s})$ is required to transition from LEO to SEL2. Consequently, we baselined a design with construction at the Earth-moon L2 (EML2) 
point. This location is desirable for the following reasons: (1) it has an illumination and viewing environment very similar to SEL2 when the moon is on the anti-sun side of the Earth, (2) an EML2 orbit provides low energy access to and from SEL2, so that the TMST can be transported to SEL2 after construction and returneded to EML2 for major servicing, (3) an EML2 orbit is close enough to the moon that it has been and continues to be under consideration for creating a crewed outpost to support the VSE. This facilitates having astronauts to support robotic construction of the TMST. Even if the crewed outpost were eventually located in a different lunar orbit where the TMST construction would take place, it should still take little time or energy to move the TMST between the construction orbit and EML2 for testing. Other advantages of EML2 is that the orbit can be maintained with a modest $\Delta \mathrm{V} / \Delta t \sim 100 \mathrm{~m} / \mathrm{sec} / \mathrm{yr}$, and can be phased to allow constant communication with the Earth and constant solar illumination (ie. no occulations).

In our design, the observatory is assembled at EML2 and tested there before being transported (by a tug or by its own propulsion) to SEL2 for normal operations. Routine maintenance, such as replacing instruments, could be performed at SEL 2 by robots. Complicated repairs would require that TMST be returned to the facilities located in its assembly orbit. D. Folta (NASA/GSFC) has modeled the flight dynamics of transporting TMST between EML2 and SEL2, and found acceptable orbits (shown in Figure 5).

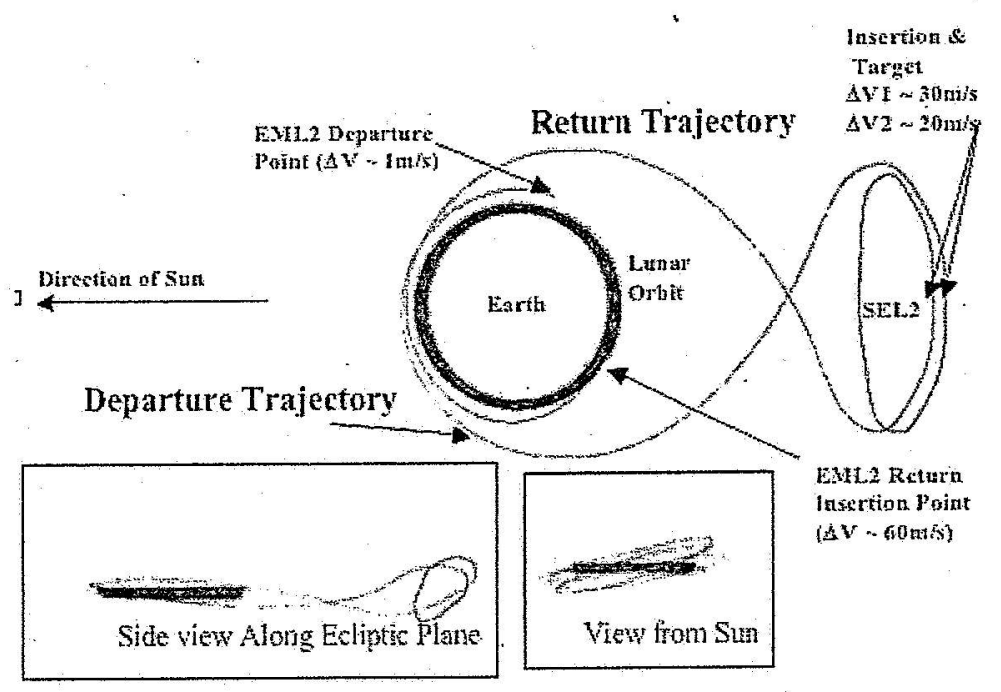

Fig 5. Orbit transition from EML2 to SEL2.

We envision that an expendable or reusable launch vehicle (ELV or RLV) will transport the observatory pieces to the moon in a highly elliptical orbit. Once in the vicinity of the moon, it will rendevous with a space tug that will then take the cargo and transport it the rest of the way to EML2, allowing the LV to continue in its orbit and return to the earth (see Figure 6). 


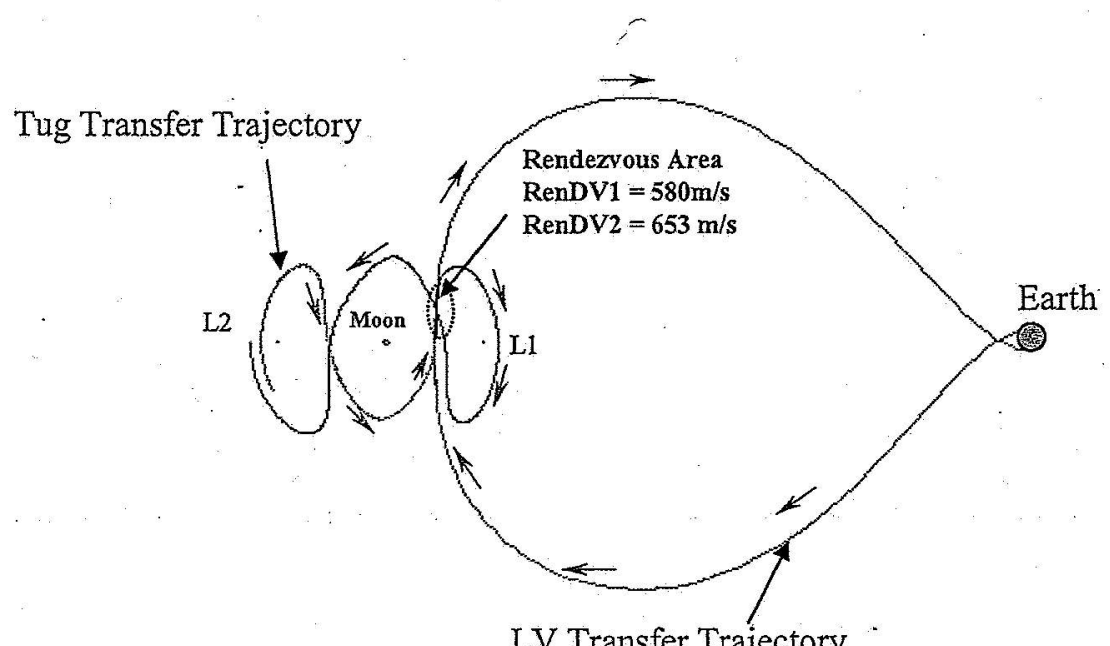

LV Transfer Trajectory

Fig 6. Schematic showing transfer of TMST parts on a reusable launch vehicle from Earth to EML1, rendevous with a space tug, and delivery of cargo to EML2.

\section{ASSEMBLY}

We now lay out a possible approach for assembly of TMST, including packaging in the launch vehicle (LV), insertion into the orbit for assembly, and the approach for construction and servicing. As part of the VSE infrastructure, we assume that there will be an assembly station near the moon. The baseline design is that TMST will be assembled by robots, but astronauts will normally be present to monitor the process and to intervene as needed. An initial goal is that the assembly process should require no more than 3 years of constant work by robots. Within reasonable limits, any assembly speed can be achieved either by developing faster robots or by having more robots work in parallel.

The $30 \mathrm{~m}$ observatory is designed to be launched as three separate elements (a 10-15m aperture telescope could be launched in one trip). Assembly begins immediately upon launch of the first element, which contains the bus and the assembly robots if they are not already located at the assembly platform. The initial assembly takes place in full sun. The second element contains the sunshade and initial telescope elements. This assembly also takes place primarily in full sun. The third element contains the OTA truss and the mirror segments. These elements are stored and assembled within the sunshade, providing a more controlled thermal environment for precision assembly.

\subsection{Launch Vehicle}

We employ the Cargo Launch Vehicle (CaLV), as defined for the VSE, as our heavy lift vehicle. The CaLV has a lift capacity of $\sim 100$ metric tons to low-earth orbit, and a fairing diameter of $\sim 8$ meters. The CaLV delivers its cargo to the assembly orbit around the moon. Although the trajectory intersects the EML2 orbit, it does not match the EML2 velocity, and will just return to earth. A space tug, which is considered to be part of the lunar outpost infrastructure, will perform a rendezvous and docking with the CaLV and then transfer the CaLV cargo to the outpost at EML2. The reason for this "handoff" scenario is twofold: (1) the CaLV payload would have to be substantially reduced to permit an upper stage that could perform an EML2 orbit insertion, and (2) this return-to-Earth LV trajectory facilitates the possibility of eventually using an RLV for this function.

\subsection{Launch Configuration}

The TMST components and assembly/servicing robots can be packaged into three, $\varnothing 7.5 \mathrm{~m} \times 14 \mathrm{~m}$ long carriers for launch. The layouts were designed for optimal packing, not sequential un-packing and assembly. This minimizes the 
number of launches, but introduces additional steps in the assembly. Each carrier is shown below. The carriers themselves are utilized as structural elements in the observatory.

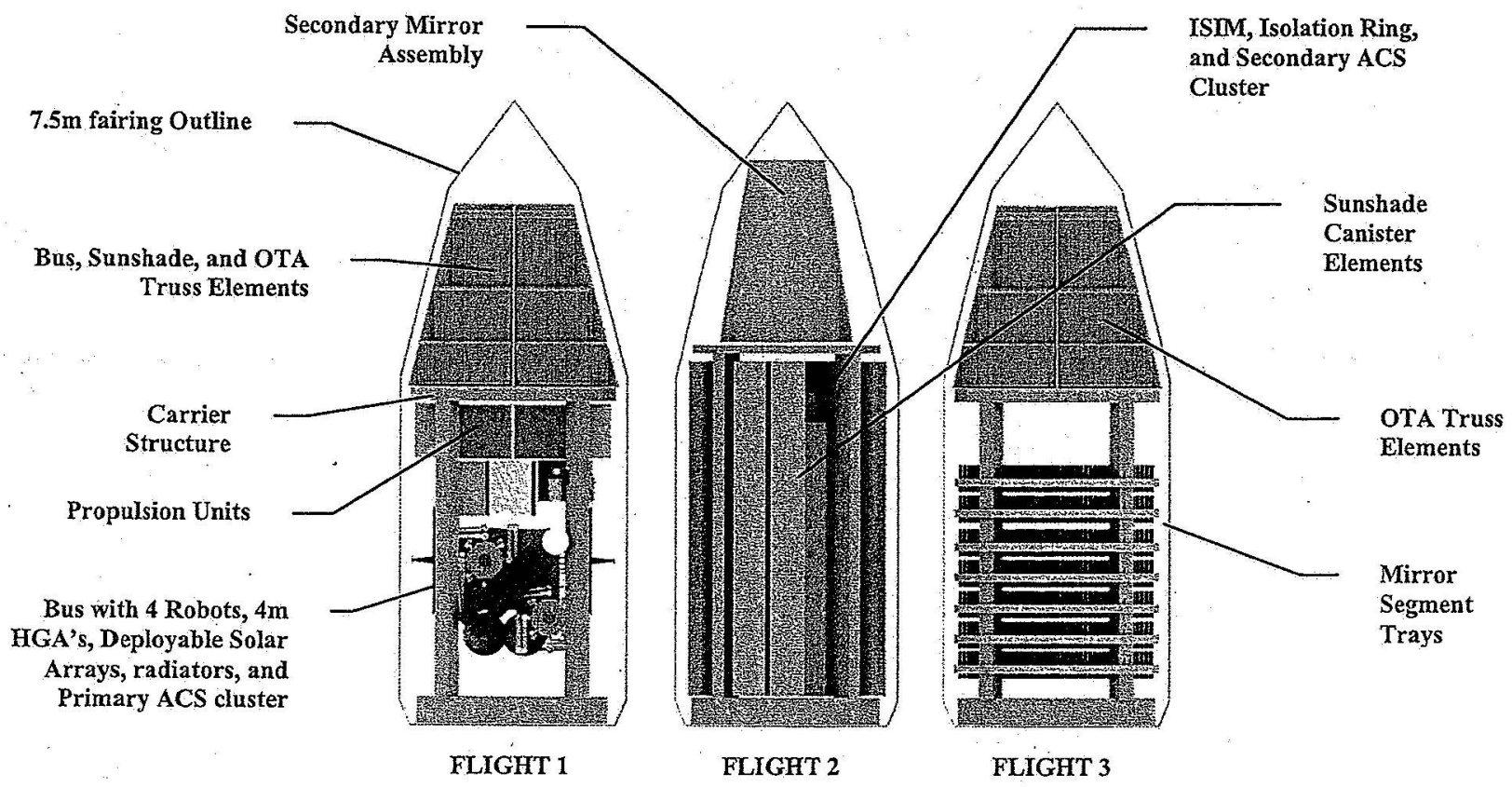

Fig 7. Packaging of TMST into three heavy lift Cargo launch vehicles.

The mirrors are packaged on trays containing $~ 196$ segments each as shown in Figure 8.

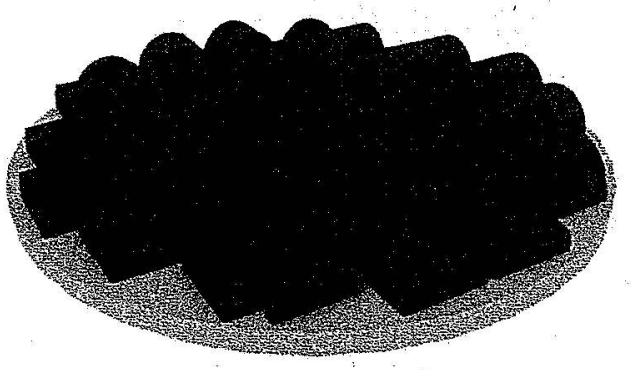

Fig 8. 196 mirror segments packaged on a launch tray.

\subsection{On-Orbit Robotic Assembly \& Testing}

A concept for assembly robotics was generated using lessons learned from the several HST servicing missions and design work done for a proposed HST Robotic Servicing Mission (HSRM). The current generation of in-space robotics, the Shuttle and ISS remote manipulation systems, as well as the upcoming Special Purpose Dexterous Manipulator (SPDM) serve as the basis for our robot. Specific issues identified in the design and testing of the HRSM verified the need for the following features: robust force-moment sensing and control, the importance of semi-autonomy, thermal control for continuous operations, power usage during robotic operations, the utility of optical targets, the need for several manipulators to simplify task accomplishment, the need for specialized tools and end effectors, and the use of "caddies". Caddies are work trays that contain all of the hardware required for a specific assembly task. 
Robotics make possible in-space assembly required for such a large structure. The robots can operate autonomously, semi-autonomously with supervision, and telerobotically. The robots are multi-armed bodies at the end of $18 \mathrm{~m}$ walking crane arms. The walking crane arm has 7 degrees of freedom (DOF) and is kinematically similar to the SRMS and SSRMS systems in use today. The assembly \& servicing robot is analogous to the SPDM, or "Dexter". The scope and scale of the activity may require the operations to be conducted in proximity to a facility capable of providing support to the long-term robotic operations. Such a facility could provide temporary staging and structural support to the launch elements during unpacking, diagnostic and test equipment to support in-process verifications, and low-latency supervision by human operators to address situations that the assembly robots cannot resolve autonomously.

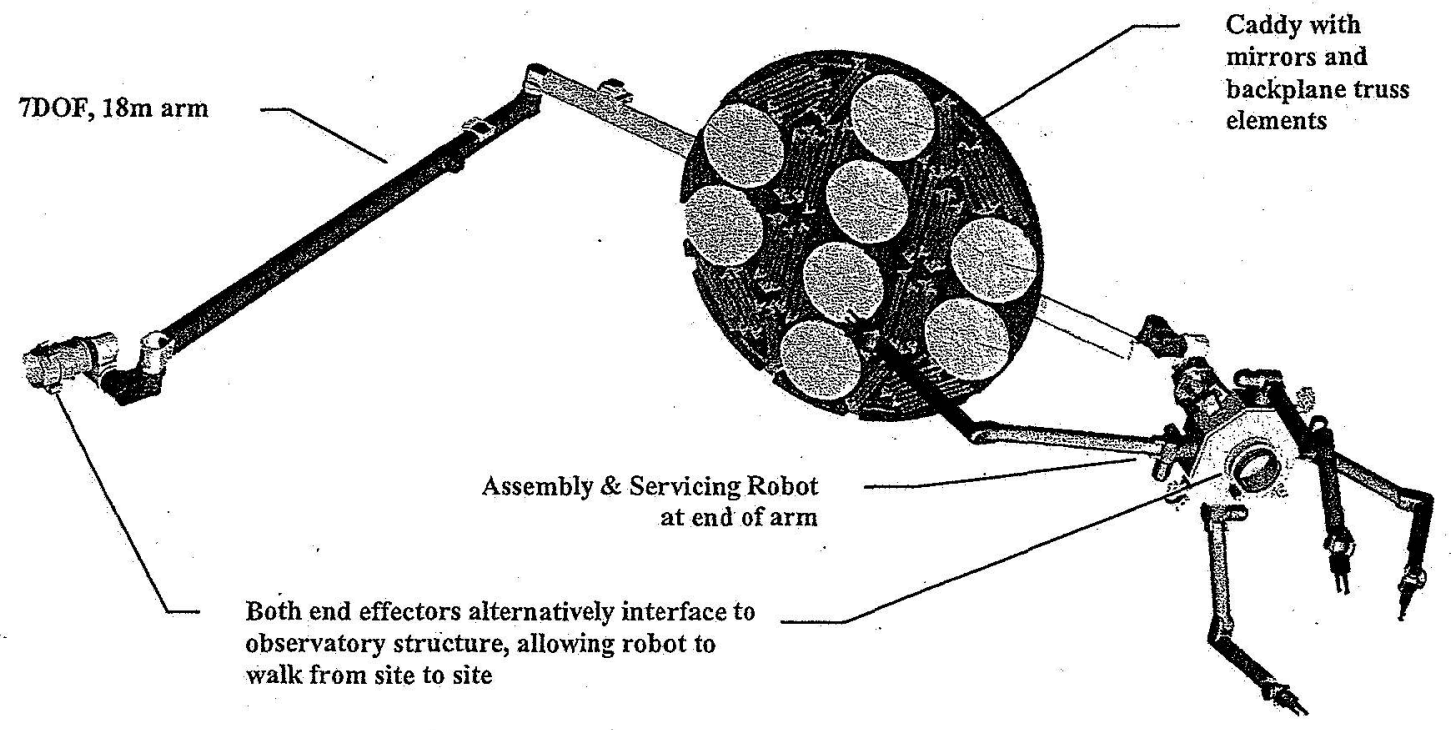

Fig 9. The Assembly and servicing robot.

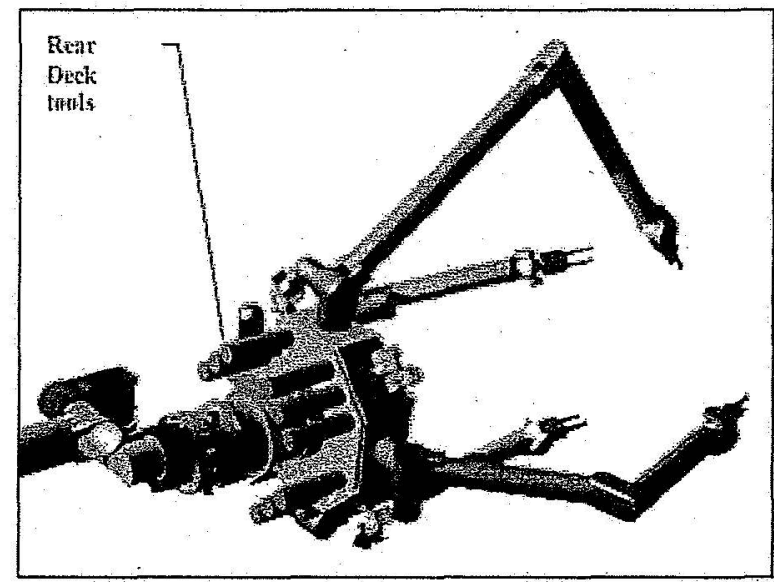

Fig 10. The robot has four arms for concurrent stabilization and manipulations. Storage for multiple tools and end effectors are located on the rear deck of the robot. 
An ordered sequence of steps was defined as a preliminary operational case for assembly of the structural truss and deployment of the reflector elements from the launch packages using robotic operations. A notional suite of robot primitive motions has been modeled and used to establish geometric and kinematic feasibility of the assembly operations. The sequence has been successfully animated. This modeling and analysis can be continued to identify and accommodate realistic details for robotic handling operations, telescope integration, component protection, and operations support requirements.

\subsection{Flight 1 Assembly}

The first flight element delivered is the bus assembly. 2 ISS-like solar array wings are deployed immediately, as well as $2 \varnothing 4 \mathrm{~m}$ high-gain communication antennae. The truss elements for the sunshade are also included. The assembly robots are released from their launch locks as soon as there is sufficient power available for checkout operations. There is a distributed array of power-data grapple fixtures throughout the observatory to support robotic agent operations.

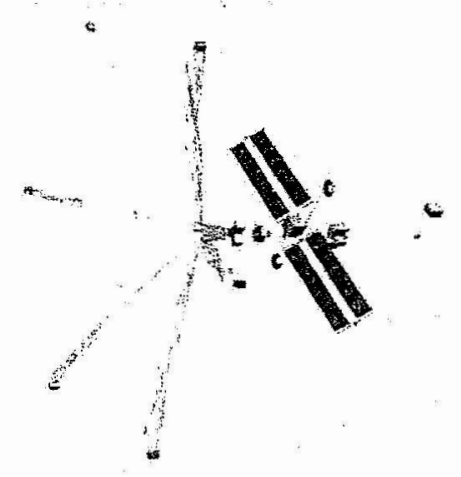

Fig 11. TMST after Flight 1 Assembly

The robots begin unpacking truss elements and assembling the base bus section. The primary propulsion modules and sunshade box are placed along this section of truss. At the other end, assembly is started on the upper bus frame. It contains the outrigger beams, outrigger tension anchors, and the ACS wheel clusters. The outrigger beams are assembled and the ACS propulsion modules placed at the ends, and tension cables rigged. The completed outrigger beams are rotated out into position, ready to support the sunshade assembly when the elements arrive in Flight 2.

\subsection{Flight 2 Assembly}

The second flight contains the sunshade frame, the sunshade canisters, the integrated secondary assembly, and the ISIM. After assembly of the sunshade frame (delivered in Flight 1), the sunshade is pulled out of its canisters like a window blind. There are 12 canisters; two for each side of the hexagonal sunshade. The ISIM and integrated secondary assembly are placed in temporary storage locations within the sunshade.
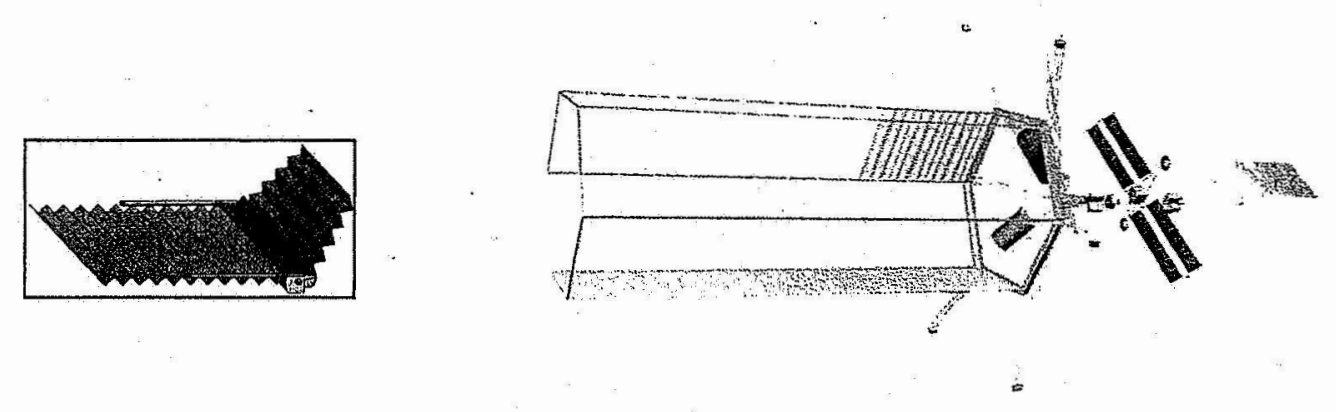

Fig 12. TMST During Flight 2 Assembly, deploying a sunshade panel 


\subsection{Flight 3 Assembly}

The OTA components, including mirrors, are delivered during flight 3. The Integrated Science Instrument Module (ISIM), delivered in Flight 2, serves as the metering ring around which the entire OTA is assembled. The OTA is assembled from the inside out. The interior axial sections of the backplane trusses are built around the ISIM, and the metering tower is then fully assembled and the secondary assembly installed.

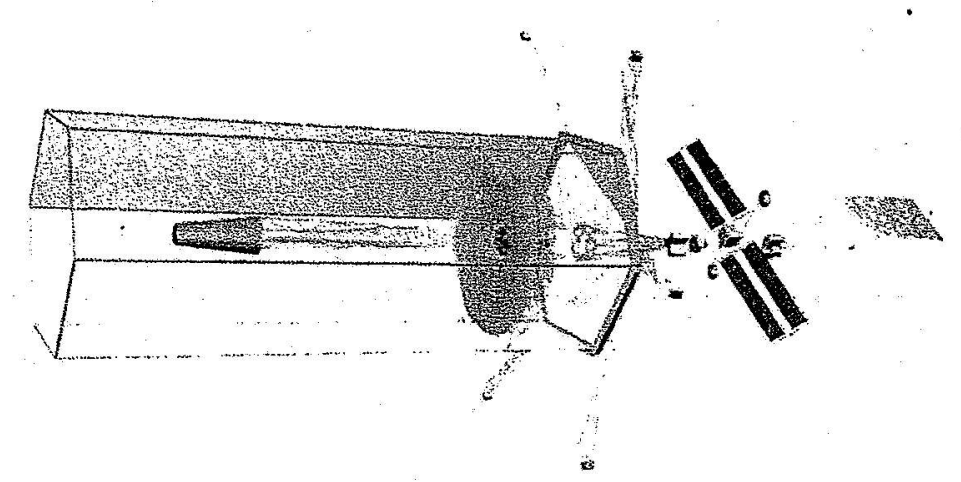

Fig. 13. TMST During Flight 3 Assembly, with partially completed primary mirror

The backplane truss and mirror segments are then assembled around the OTA core, methodically assembling the backplane and populating it with mirror segments ring by ring from the inside out. As each segment is placed, the filled diameter grows until the full diameter is reached. Each segment could be phased shortly after installation. This would allow the observatory to conduct reduced-aperture engineering or science observations at any time during primary mirror population.

\subsection{OTA Alignment and Testing}

The OTA system is designed to be aligned automatically on-orbit using imagery from several wavefront cameras and laser-truss metrology. Since the ISIM, which contains the wavefront cameras required for segment alignment, is put in place prior to the primary mirror, individual segment alignment may occur as they are assembled. Ground testing of such a large system would be far too expensive. Also, designing the OTA to be ground-testable would incur mass and performance penalties that would result in greatly increased costs.

\subsection{On-Orbit Robotic Servicing}

Servicing is required to realize the full benefit of the large investment in assembly and commissioning of the TMST. The overall design for assembly inherently accommodates servicing by virtue of the modular nature of the components. Every component of the observatory is designed to be robotically replaced, in order to achieve a 100 year lifetime. A five-year servicing cycle is taken as a working baseline concept, and the station-keeping propulsion resource has been sized for this interval. This need for regular visits for resupply of consumables sets the cadence for repair and upgrade opportunities. Each servicing mission is presumed to carry a full set of propulsion modules for the TMST, and a partial replacement of power system components, i.e., solar array segments and batteries. The servicing missions also provide non-routine upgrade of selected rapidly-advancing technology items, such as science instruments, data and communications avionics, and any needed repair of degraded components, such as mechanisms, surface treatment for exposed materials, radiation- or meteorite-damaged items. Every subsystem, including the principal and secondary structures and utility distribution systems, incorporates modularity and distributed functionality such that any element can be replaced for repair or upgrade without critically disabling the performance and functionality of the entire observatory as a whole. The resident robotic servicing agents will also be repaired and upgraded. Between servicing missions they are stored in a quiescent dormant state, but ready for use if called into service to address some eventuality within their capability, i.e., inspection, adjustment, cleaning, clearing of faults, etc. The visiting servicer vehicle may be based at a logistics node that supports other space-faring traffic, sharing the investment in infrastructure across a broad market of users over an extended period of service. The operations at the logistics node can prepare the servicer, loading mission-specific packages launched from Earth or generic consumables and other items from an inventory accumulated over time for support of multiple standard users having a compatible modular component architecture. 
If a major overhaul of the TMST is ever warranted, say to install a completely new technology or mission functionality, or repair some major fault which cannot be effectively addressed by the resident robotic agents, the entire TMST could be moved to the vicinity of a servicing site, such as an Earth-moon libration point. The servicer vehicle visiting TMST would in this case carry sufficient fuel to serve as a tug capable of a round-trip from the depot to the TMST site and return with TMST. Another tug sortie would return TMST to its operational orbit, or TMST could make the transfer using on-board resources as in its initial deployment.

Propulsion unit refill/replace and instrument changeout will likely be the most common operations. These operations occur "on site" in the science orbit at approximately 5 year intervals. Advances in instrument technology, particularly detectors, as well as degradation due to radiation, are often the reason for frequent upgrades. In our design, the instruments occupy fully replaceable units within the ISIM, and an instrument change would be "plug and play" and performed robotically. The instruments are sized such that 4 can be launched in existing EELV $5 \mathrm{~m}$ fairings. A typical servicing payload would include 1-4 instruments and replacement propulsion packages.

\section{CONCLUSIONS}

We have performed a preliminary study of the design of a large (30 meter aperture) UVOIR space telescope and its robotic assembly in deep space (in lunar orbit). Such a design is made possible only by heavy-lift launch vehicles and infrastructure in orbit around the moon, as planned in the VSE several decades from now. Given such an infrastructure, and capable robots, our design for TMST does not appear to present any significant technological hurdles. The optics, structure, wavefront sensing, attitude control and other spacecraft elements all appear to be well within current reach. Identified work needed to complete this preliminary design include: (1) designs for element handling and coupling interfaces, (2) parameterization of robotic tasks such as speed of operation, sensing, automation, (3) robotic handling requirements such as force and torque reaction, (4) command and control of robotic tasks, and (5) engineering model evaluation of items 1-4. In addition, further work is needed to define the OTA phasing/alignment architecture, mechanical stability, vibration isolation, pointing control and jitter analysis.

We thank Dave Folta for flight dynamics support, and the staff of GSFC's Integrated Design Center during a 10 day engineering study of TMST in 2005.

\section{REFERENCES}

1. Illingworth, G. and Kennicutt, R., in preface to "Hubble's Science Legacy: Future Optical/Ultraviolet Astronomy from Space", 2003, ed. Sembach, K., Blades, C., Illingworth, G. and Kennicutt, R., ASP Conf Series 291, Proc. of conference held at University of Chicago, 2002.

2. Nella, , J., et al. "James Webb Space Telescope (JWST) Observatory Architecture and Performance", AIAA2004-5896

3. Illingworth, G. et al, 2003, proposal to NASA for Vision Missions.

4. The Vision for Space Exploration, 2004, http://www nasa.gov/pdf/55583main vision space exploration2.pdf

5. Muller, R., "Robotic Assembly of a 20-Meter Space Telescope With Extension to 40 Meter Space Telescopes", QSS Group, Inc., Lanham, MD, 2000

6. Lille, C.F., "Large Deployable Telescopes for Future Space Observatories", 2005, Proc SPIE, Vol. 5899, 108.

7. Kilston, S., Bally, J., "Potential Paths in Space Astronomy Over the Next 50 Years", Proc SPIE, Vol. 4835

8. J. Green et al "The Modern Universe Space Telescope (MUST)", a Vision Mission Concept Study Report delivered to NASA HQ, 2006.

9. Stevens, J., King, D., "Leveraging Exploration Capabilities for Space-Based Astronomical Observatories", Proc SPIE Vol. 5899

10. Friedman, E., "The Technical Path to In-Space Testing of Large Optics", Proc SPIE Vol. 5899

11. Lester, D. \& Lillie, C. "Servicing the Single Aperture Far Infrared (SAFIR) Telescope from a LunarExploration Enabled Gateway", 2005, Space Resources Roundtable VII: LEAG Conference on Lunar Exploration, LPI Contribution No. 1287, p.59

12. Basu, S. "Conceptual design of an autonomously assembled space telescope", 2004,Proc SPIE, 5166, 98 